\title{
Pulsating strings with mixed three-form flux
}

\author{
Rafael Hernández, Juan Miguel Nieto and Roberto Ruiz \\ Departamento de Física Teórica I, Universidad Complutense de Madrid, \\ Madrid, 28040 Spain \\ E-mail: rafael.hernandez@is.ucm.es, juanieto@ucm.es, roruiz@ucm.es
}

AbStract: Circular strings pulsating in $A d S_{3} \times S^{3} \times T^{4}$ with mixed R-R and NS-NS three-form fluxes can be described by an integrable deformation of the one-dimensional Neumann-Rosochatius mechanical model. In this article we find a general class of pulsating solutions to this integrable system that can be expressed in terms of elliptic functions. In the limit of strings moving in $A d S_{3}$ with pure NS-NS three-form flux, where the action reduces to the $\operatorname{SL}(2, \mathbb{R})$ WZW model, we find agreement with the analysis of the classical solutions of the system performed using spectral flow by Maldacena and Ooguri. We use our elliptic solutions in $A d S_{3}$ to extend the dispersion relation beyond the limit of pure NS-NS flux.

KEYwords: AdS-CFT Correspondence, Gauge-gravity correspondence

ArXiv EPRINT: 1803.03078

The integrable structure that underlies the $\mathrm{AdS}_{3} / \mathrm{CFT}_{2}$ correspondence has provided a deep understanding of numerous aspects of string theory in backgrounds with an $A d S_{3}$ factor and two-dimensional conformal field theories with maximal supersymmetry [1]-[22]. Integrability has also been shown to remain a symmetry of general string backgrounds that support a mixture of R-R and NS-NS three-form fluxes [23]. This discovery has led to an insightful view of many features of the $\mathrm{AdS}_{3} / \mathrm{CFT}_{2}$ correspondence in the presence of mixed fluxes [24-26]-[42]. One of these developments was the demonstration that the sigma-model of type IIB closed strings spinning in $A d S_{3} \times S^{3}$ with mixed fluxes corresponds to an integrable deformation of the Neumann-Rosochatius mechanical system [43, 44]. The identification of the Lagrangian that describes spinning strings with mixed fluxes with a deformation of the Neumann-Rosochatius system allows the use of a systematic approach to the construction of general classes of solutions. In this letter we will extend the problem to the case of an ansatz corresponding to closed strings pulsating in $A d S_{3} \times S^{3}$. In particular, we will show that pulsating strings with mixed fluxes can also be treated using the integrable 
deformation of the Neumann-Rosochatius system obtained from the spinning string ansatz. We will make use of the flux-deformation of the Uhlenbeck constants of the model to integrate the equations of motion in terms of Jacobi elliptic functions. We will also study the problem in the limit of pure NS-NS three-form flux, where the $A d S_{3}$ piece of the system reduces to the $\mathrm{SL}(2, \mathbb{R})$ WZW model. On the basis of our class of elliptic solutions we will derive a general form of the dispersion relation valid beyond the WZW point.

In what follows we will first present the Neumann-Rosochatius system that arises from the motion of closed strings pulsating in $A d S_{3} \times S^{3} \times T^{4}$ with non-vanishing NS-NS flux. We will consider no dynamics along the torus, and thus the background metric will be

$$
d s^{2}=-\cosh ^{2} \rho d t^{2}+d \rho^{2}+\sinh ^{2} \rho d \phi^{2}+d \theta^{2}+\sin ^{2} \theta d \phi_{1}^{2}+\cos ^{2} \theta d \phi_{2}^{2},
$$

together with

$$
b_{t \phi}=q \sinh ^{2} \rho, \quad b_{\phi_{1} \phi_{2}}=-q \cos ^{2} \theta,
$$

for the NS-NS B-field, where $0 \leq q \leq 1$. The limit $q=0$ corresponds to the case of pure $\mathrm{R}-\mathrm{R}$ flux, while setting $q=1$ we are left with pure NS-NS flux. In the case of pure R-R flux the sigma-model for closed strings rotating in $A d S_{3} \times S^{3}$ reduces to the NeumannRosochatius system $[45,46]$. The presence of the NS-NS flux term leads to an integrable deformation of the Neumann-Rosochatius model $[43,44]$. In this letter we will extend the analysis in that reference for the spinning string ansatz to the case of pulsating strings. In order to introduce the pulsating ansatz it will be convenient to use the embedding coordinates of $A d S_{3}$ and $S^{3}$, which are related to the global angles by

$$
\begin{aligned}
Y_{1}+i Y_{2} & =\sinh \rho e^{i \phi}, \\
X_{1}+i X_{2} & =\sin \theta e^{i \phi_{1}}, \quad Y_{3}+i Y_{0}=\cosh \rho e^{i t}, \\
X_{4} & =\cos \theta e^{i \phi_{2}} .
\end{aligned}
$$

In these coordinates the ansatz for a pulsating string is

$$
\begin{aligned}
Y_{1}+i Y_{2} & =z_{1}(\tau) e^{i \beta_{1}(\tau)+i k_{1} \sigma}, \\
X_{1}+i X_{2} & =r_{1}(\tau) e^{i \alpha_{1}(\tau)+i m_{1} \sigma}, \quad Y_{3}+i Y_{0}=z_{0}(\tau) e^{i \beta_{0}(\tau)}, \\
X_{3}+i X_{4} & =r_{2}(\tau) e^{i \alpha_{2}(\tau)+i m_{2} \sigma},
\end{aligned}
$$

where we have excluded the winding along the time coordinate because the time direction has to be single-valued. When we enter this ansatz in the world-sheet action in the conformal gauge we find

$$
\begin{aligned}
L= & \frac{\sqrt{\lambda}}{2 \pi}\left[\sum_{i=1}^{2} \frac{1}{2}\left(\dot{r}_{i}^{2}+r_{i}^{2} \dot{\alpha}_{i}^{2}-r_{i}^{2} m_{i}^{2}\right)+q r_{2}^{2}\left(m_{2} \dot{\alpha}_{1}-m_{1} \dot{\alpha}_{2}\right)-\frac{\Lambda}{2}\left(r_{1}^{2}+r_{2}^{2}-1\right)\right. \\
& \left.+\frac{1}{2} g^{a b}\left(\dot{z}_{a} \dot{z}_{b}+z_{a} z_{a} \dot{\beta}_{b}^{2}\right)-z_{1}^{2} k_{1}^{2}-q k_{1} z_{1}^{2} \dot{\beta}_{0}-\frac{\tilde{\Lambda}}{2}\left(g^{a b} z_{a} z_{b}+1\right)\right],
\end{aligned}
$$

where the dot stands for derivatives with respect to $\tau$, the Lagrange multipliers $\Lambda$ and $\tilde{\Lambda}$ are needed to impose that the solutions lie, respectively, on $S^{3}$ and $A d S_{3}$, and we have taken $g=\operatorname{diag}(-1,1)$, with $a=0,1$. We must note that the flux term in (7) appears with the 
opposite sign than that in the Lagrangian coming from the spinning string ansatz [43, 44]. The equations of motion for the radial coordinates following from (7) are given by

$$
\begin{aligned}
& \ddot{r}_{1}=-r_{1} m_{1}^{2}+r_{1} \dot{\alpha}_{1}^{2}-\Lambda r_{1}, \\
& \ddot{r}_{2}=-r_{2} m_{2}^{2}+r_{2} \dot{\alpha}_{2}^{2}-\Lambda r_{2}+2 q r_{2}\left(m_{2} \dot{\alpha}_{1}-m_{1} \dot{\alpha}_{2}\right),
\end{aligned}
$$

for the $r_{i}$ coordinates, while for the $z_{a}$ coordinates they are

$$
\begin{aligned}
& \ddot{z}_{0}=z_{0} \dot{\beta}_{0}^{2}-\tilde{\Lambda} z_{0}, \\
& \ddot{z}_{1}=z_{1} \dot{\beta}_{1}^{2}-z_{1} k_{1}^{2}-\tilde{\Lambda} z_{1}-2 q z_{1} k_{1} \dot{\beta}_{0} .
\end{aligned}
$$

The cyclic nature of the spherical and hyperbolic angular coordinates in the Lagrangian implies that its equations of motion can be easily integrated once,

$$
\begin{array}{ll}
\dot{\alpha}_{1}=\frac{v_{1}-q r_{2}^{2} m_{2}}{r_{1}^{2}}, & \dot{\alpha}_{2}=\frac{v_{2}+q r_{2}^{2} m_{1}}{r_{2}^{2}}, \\
\dot{\beta}_{0}=-\frac{u_{0}+q k_{1} z_{1}^{2}}{z_{0}^{2}}, & \dot{\beta}_{1}=\frac{u_{1}}{z_{1}^{2}},
\end{array}
$$

where $v_{i}$ and $u_{a}$ are some integration constants. We can use equations (12) and (13) to write the energy, the Lorentzian spin and the two angular momenta of the string as

$$
E=-\sqrt{\lambda} u_{0}, \quad S=\sqrt{\lambda} u_{1}, \quad J_{1}=\sqrt{\lambda} v_{1}, \quad J_{2}=\sqrt{\lambda} v_{2} .
$$

The equations of motion coming from (7) must be supplied with the Virasoro constraints, which are responsible for the coupling between the $A d S_{3}$ and the $S^{3}$ pieces of the system. They take the form

$$
\begin{array}{r}
\sum_{i=1}^{2}\left[\dot{r}_{i}^{2}+\left(\dot{\alpha}_{i}^{2}+m_{i}^{2}\right) r_{i}^{2}\right]+\sum_{i=1}^{2} g^{i i}\left(\dot{z}_{i}^{2}+z_{i}^{2} \dot{\beta}_{i}^{2}\right)+k_{1}^{2} z_{1}^{2}=0 \\
z_{1}^{2} k_{1} \dot{\beta}_{1}+\sum_{i=1}^{2} r_{i}^{2} m_{i} \dot{\alpha}_{i}=0 .
\end{array}
$$

We will now move to the construction of general solutions to the above system. In order to proceed, we will follow $[45,46]$ and introduce ellipsoidal coordinates $\zeta$ and $\mu$ for the sphere and the Anti-de Sitter factors defined, respectively, as the roots of the equations

$$
\frac{r_{1}^{2}}{\zeta-m_{1}^{2}}+\frac{r_{2}^{2}}{\zeta-m_{2}^{2}}=0, \quad \frac{z_{1}^{2}}{\mu-k_{1}^{2}}-\frac{z_{0}^{2}}{\mu}=0 .
$$

The ranges of the ellipsoidal coordinates are $m_{1}^{2} \leq \zeta \leq m_{2}^{2}$ and $k_{1}^{2} \leq \mu$. When we enter directly $\zeta$ and $\mu$ into the equations of motion for $r_{i}$ and $z_{a}$ we are left with a second order differential equation for each ellipsoidal coordinate. However, we can also use the Uhlenbeck constants of the system to reduce the problem to a pair of independent first order differential equations $[45,46]$. The Uhlenbeck constants for the pulsating NeumannRosochatius system in the presence of the flux deformation can be obtained immediately 
from the ones for the spinning Neumann-Rosochatius system in $[43,44]$ just by replacing $q$ by $-q$, because of the change in the sign of flux terms. Thus the Uhlenbeck constants corresponding to motion of the pulsating string either on the sphere or on the Anti-de Sitter factor are, respectively, given by ${ }^{1}$

$$
\begin{aligned}
& \bar{I}_{1}=r_{1}^{2}\left(1-q^{2}\right)+\frac{1}{m_{1}^{2}-m_{2}^{2}}\left[\left(r_{1} \dot{r}_{2}-\dot{r}_{1} r_{2}\right)^{2}+\frac{\left(v_{1}-q \omega_{2}\right)^{2}}{r_{1}^{2}} r_{2}^{2}+\frac{v_{2}^{2}}{r_{2}^{2}} r_{1}^{2}\right], \\
& \bar{F}_{1}=z_{1}^{2}\left(1-q^{2}\right)+\frac{1}{k_{1}^{2}}\left[\left(z_{1} \dot{z}_{0}-\dot{z}_{1} z_{0}\right)^{2}+\frac{\left(u_{0}-q k_{1}\right)^{2}}{z_{0}^{2}} z_{1}^{2}+\frac{u_{1}^{2}}{z_{1}^{2}} z_{0}^{2}\right] .
\end{aligned}
$$

If we write the Uhlenbeck integrals in terms of the ellipsoidal coordinates we conclude that

$$
\dot{\zeta}^{2}=-4 P_{3}(\zeta), \quad \dot{\mu}^{2}=-4 Q_{3}(\zeta),
$$

where the third order polynomials $P_{3}(\zeta)$ and $Q_{3}(\zeta)$ are given by

$$
\begin{aligned}
P_{3}(\zeta)= & -\left(1-q^{2}\right)\left(m_{2}^{2}-\zeta\right)\left(\zeta-m_{1}^{2}\right)^{2}+\bar{I}_{1}\left(m_{2}^{2}-m_{1}^{2}\right)\left(m_{2}^{2}-\zeta\right)\left(\zeta-m_{1}^{2}\right) \\
& +\left(v_{1}-q m_{2}\right)^{2}\left(m_{2}^{2}-\zeta\right)^{2}+v_{2}^{2}\left(\zeta-m_{1}^{2}\right)^{2} \equiv\left(1-q^{2}\right) \prod_{i=1}^{3}\left(\zeta-\zeta_{i}\right) \\
Q_{3}(\mu)= & \left(1-q^{2}\right)\left(\mu-k_{1}^{2}\right)^{2} \mu-\bar{F}_{1} k_{1}^{2}\left(\mu-k_{1}^{2}\right) \mu+\left(u_{0}-q k_{1}\right)^{2}\left(\mu-k_{1}^{2}\right)^{2}+u_{1}^{2} \mu^{2} \\
\equiv & \left(1-q^{2}\right) \prod_{i=1}^{3}\left(\mu-\mu_{i}\right) .
\end{aligned}
$$

The solution to equations (20) can be written in terms of the Jacobian elliptic functions (see $[43,44]$ for details in the case of the spinning string ansatz). We find

$$
\begin{aligned}
& \zeta(\tau)=\zeta_{3}+\left(\zeta_{2}-\zeta_{3}\right) \operatorname{sn}^{2}\left(\sqrt{\left(1-q^{2}\right)\left(\zeta_{3}-\zeta_{1}\right)} \tau+\tau_{0}, \kappa\right), \\
& \mu(\tau)=\mu_{2}+\frac{\left(\mu_{3}-\mu_{2}\right)\left(\mu_{2}-\mu_{1}\right)}{\mu_{3}-\mu_{1}} \operatorname{sd}^{2}\left(\sqrt{\left(1-q^{2}\right)\left(\mu_{3}-\mu_{1}\right)} \tau+\tau_{0}^{\prime}, \nu\right),
\end{aligned}
$$

where $\tau_{0}$ and $\tau_{0}^{\prime}$ are integration constants that can be set to zero by performing a rotation, and the elliptic moduli are

$$
\kappa=\frac{\zeta_{3}-\zeta_{2}}{\zeta_{3}-\zeta_{1}}, \quad \nu=\frac{\mu_{3}-\mu_{2}}{\mu_{3}-\mu_{1}}
$$

We therefore conclude that ${ }^{2}$

$$
\begin{aligned}
& r_{1}^{2}(\tau)=\frac{\zeta_{3}-m_{1}^{2}}{m_{2}^{2}-m_{1}^{2}}-\frac{\zeta_{3}-\zeta_{2}}{m_{2}^{2}-m_{1}^{2}} \operatorname{sn}^{2}\left(\sqrt{\left(1-q^{2}\right)\left(\zeta_{3}-\zeta_{1}\right)} \tau, \kappa\right) \\
& z_{1}^{2}(\tau)=\frac{\mu_{2}-k_{1}^{2}}{k_{1}^{2}}+\frac{\left(\mu_{3}-\mu_{2}\right)\left(\mu_{2}-\mu_{1}\right)}{k_{1}^{2}\left(\mu_{3}-\mu_{1}\right)} \mathrm{sd}^{2}\left(\sqrt{\left(1-q^{2}\right)\left(\mu_{3}-\mu_{1}\right)} \tau, \nu\right) .
\end{aligned}
$$

\footnotetext{
${ }^{1}$ The integrability of the Neumann-Rosochatius system follows from the existence of a set of integrals of motion in involution, the Uhlenbeck constants [47]. In the case of strings spinning in $S^{3}$ there are two integrals $I_{1}$ and $I_{2}$, constrained to satisfy $I_{1}+I_{2}=1$. In the presence of flux they are deformed to $\bar{I}_{1}$ and $\bar{I}_{2}$, with the condition $\bar{I}_{1}+\bar{I}_{2}=1[43,44]$. A similar set of constants, $F_{0}$ and $F_{1}$, with the constraint $F_{1}-F_{0}=-1$, and their corresponding deformation by the flux term, arises when the string spins in $A d S_{3}$.

${ }^{2}$ Pulsating string solutions in $A d S_{3} \times S^{3}$ with mixed R-R and NS-NS fluxes have been considered before in [37]. To contact with the notation in that reference we just need to identify the roots $R_{+}$and $R_{-}$in there with our choice of roots through $R_{+}=\mu_{3} / k_{1}^{2}-1$ and $R_{-}=\mu_{1} / k_{1}^{2}-1$.
} 
We must stress that we need to order the roots of the ellipsoidal coordinate $\zeta$ for the sphere in such a way that $\zeta_{1}<\zeta_{3}$. On the contrary, the ellipsoidal coordinate $\mu$ for the Anti-de Sitter factor is unbounded and symmetrical under permutation of the roots $\mu_{1}$ and $\mu_{3}$ and thus there is no need to choose the roots $\mu_{1}$ and $\mu_{3}$ according to any particular ordering. Moreover, we should choose $\mu_{2} \geq k_{1}^{2}$ because $z_{1}^{2} \geq 0$. Furthermore, we will restrict the elliptic moduli (25) to their fundamental domains $0 \leq \kappa, \tau \leq 1$, which implies that $\zeta_{1}<\zeta_{2}<\zeta_{3}$, and either $\mu_{1}<\mu_{2}<\mu_{3}$ or $\mu_{3}<\mu_{2}<\mu_{1}$.

We will now focus on the analysis of string solutions restricted to pulsate in $A d S_{3} \times S^{1}$ in the limit of pure NS-NS three-form flux. We will therefore set $r_{1}=\alpha_{1}=0$, and $r_{2}=1$ and $\alpha_{2}=\omega$. We must first note that the cubic term in $Q_{3}(\mu)$ is dressed with a factor $1-q^{2}$. Thus in the case of pure NS-NS flux the degree of the polynomial reduces to two, and the solution can be written in terms of trigonometric functions. In order to understand the reduction of the problem from the point of view of the roots of the polynomial, we will first present them for general values of $q$. The Virasoro constraints reduce now to

$$
\begin{aligned}
\dot{z}_{0}^{2}+\frac{\left(u_{0}+q k_{1} z_{1}^{2}\right)^{2}}{z_{0}^{2}} & =\dot{z}_{1}^{2}+k_{1}^{2} z_{1}^{2}+\omega^{2}, \\
u_{1} & =0 .
\end{aligned}
$$

Using condition (29) it is immediate to check that one of the roots of the polynomial (22) is given by $\mu_{0}=k_{1}^{2}$. The remaining roots are given by

$$
\mu_{ \pm}=\frac{f_{1}}{2}-\frac{\left(u_{0}-q k_{1}\right)^{2} \mp \sqrt{\left[\left(u_{0}-q k_{1}\right)^{2}-\left(1-q^{2}\right) f_{1}\right]^{2}+4\left(1-q^{2}\right) k_{1}^{2}\left(u_{0}-q k_{1}\right)}}{2\left(1-q^{2}\right)}
$$

where we have defined

$$
f_{1}=k_{1}^{2}+\frac{k_{1}^{2} \bar{F}_{1}}{1-q^{2}}
$$

If we exclude the case with $\bar{F}_{1}=0$, which corresponds to the trivial limit where the solution collapses to a point, it is immediate to check that the roots satisfy $\mu_{-}<\mu_{0}<\mu_{+}$. It is also clear that the limit of pure NS-NS flux depends on the sign of the term $\left(u_{0}-k_{1}^{2}\right)^{2}-k_{1}^{2} \bar{F}_{1}$. If we choose $\left(u_{0}-k_{1}^{2}\right)^{2}>k_{1}^{2} \bar{F}_{1}$, the roots become

$$
\mu_{-} \rightarrow-\infty, \quad \mu_{0} \rightarrow k_{1}^{2}, \quad \mu_{+} \rightarrow \frac{\left(u_{0}-k_{1}\right)^{2} k_{1}^{2}}{\left(u_{0}-k_{1}\right)^{2}-k_{1}^{2} \bar{F}_{1}}
$$

In this case, named short string regime in [48], the hyperbolic radius remains bounded, and the solution is given by

$$
z_{1}^{2}(\tau)=\frac{k_{1}^{2} \bar{F}_{1}}{\left(u_{0}-k_{1}\right)^{2}-k_{1}^{2} \bar{F}_{1}} \sin ^{2}\left(\sqrt{\left(u_{0}-k_{1}^{2}\right)^{2}-k_{1}^{2} \bar{F}_{1}} \tau\right) .
$$

We can therefore write $z_{1}^{2}(\tau)=\sinh ^{2} \rho(\tau)=\sinh ^{2} \rho_{0} \sin (\alpha \tau)$, so that

$$
\cosh \rho_{0}=\frac{\left|u_{0}-k_{1}\right|}{\sqrt{\left(u_{0}-k_{1}^{2}\right)^{2}-k_{1}^{2} \bar{F}_{1}}} .
$$


The parameter $\rho_{0}$ can be interpreted as the maximum hyperbolic radius that the solution can reach. On the contrary, in the case where $k_{1}^{2} \bar{F}_{1}>\left(u_{0}-k_{1}^{2}\right)^{2}$ the roots become

$$
\mu_{-} \rightarrow-\frac{\left(u_{0}-k_{1}\right)^{2} k_{1}^{2}}{k_{1}^{2} \bar{F}_{1}-\left(u_{0}-k_{1}\right)^{2}}, \quad \mu_{0} \rightarrow k_{1}^{2}, \quad \mu_{+} \rightarrow \infty
$$

and the hyperbolic radius is unbounded. This is the long string regime of [48], and the solution reduces now to

$$
z_{1}^{2}(\tau)=\frac{k_{1}^{2} \bar{F}_{1}}{k_{1}^{2} \bar{F}_{1}-\left(u_{0}-k_{1}\right)^{2}} \sinh ^{2}\left(\sqrt{k_{1}^{2} \bar{F}_{1}-\left(u_{0}-k_{1}\right)^{2}} \tau\right) .
$$

In this case we will introduce $\rho_{0}$ using $z_{1}^{2}(\tau)=\sinh ^{2} \rho(\tau)=\cosh ^{2} \rho_{0} \sinh (\alpha \tau)$. Accordingly,

$$
\sinh \rho_{0}=\frac{\left|u_{0}-k_{1}\right|}{\sqrt{k_{1}^{2} \bar{F}_{1}-\left(u_{0}-k_{1}^{2}\right)^{2}}} .
$$

Now the parameter $\rho_{0}$ does not have the interpretation of the maximum size of the solution. It is also worth to consider the threshold case, with $k_{1}^{2} \bar{F}_{1}=\left(u_{0}-k_{1}^{2}\right)^{2}$, where the ellipsoidal coordinate $\mu$ displays a parabolic behaviour. In this case both $\mu_{-}$and $\mu_{+}$diverge and

$$
z_{1}^{2}(\tau)=k_{1}^{2} \bar{F}_{1} \tau^{2}
$$

In the same way as the short and long string regimes are, respectively, constructed by means of spectral flow on time-like and space-like geodesics, the parabolic behaviour can be understood as the result of performing spectral flow on light-like geodesics.

In order to fix the sign of $\alpha$ in the previous expressions we need to find the dependence of the time coordinate $t$ on $\tau$. We can obtain that dependence by direct integration of the equation of motion (13) for $\dot{\beta}_{0}$. In the short string regime, we conclude that ${ }^{3}$

$$
\tan t(\tau)=\frac{\tan \left(-k_{1} \tau\right)+\cosh \rho_{0} \tan \left(-\operatorname{sign}\left(u_{0}-k_{1}\right) \sqrt{\left(u_{0}-k_{1}\right)^{2}-\bar{F}_{1} k_{1}^{2}} \tau\right)}{1-\cosh \rho_{0} \tan \left(-k_{1} \tau\right) \tan \left(-\operatorname{sign}\left(u_{0}-k_{1}\right) \sqrt{\left(u_{0}-k_{1}\right)^{2}-\bar{F}_{1} k_{1}^{2}} \tau\right)} .
$$

In the long string regime we can proceed identically, or continue analytically expression (39), to find that

$$
\tan t(\tau)=\frac{\tan \left(-k_{1} \tau\right)+\sinh \rho_{0} \tanh \left(-\operatorname{sign}\left(u_{0}-k_{1}\right) \sqrt{k_{1}^{2} \bar{F}_{1}-\left(u_{0}-k_{1}\right)^{2}} \tau\right)}{1-\sinh \rho_{0} \tan \left(-k_{1} \tau\right) \tanh \left(-\operatorname{sign}\left(u_{0}-k_{1}\right) \sqrt{k_{1}^{2} \bar{F}_{1}-\left(u_{0}-k_{1}\right)^{2}} \tau\right)} .
$$

This is indeed the result in [48] provided we read $\alpha=-\operatorname{sign}\left(u_{0}-k_{1}\right) \sqrt{\left|\left(u_{0}-k_{1}\right)^{2}-\bar{F}_{1} k_{1}^{2}\right|}$ and identify the winding number $k_{1}$ with $-w$ in that reference. Besides, the threshold case corresponds simply to

$$
t(\tau)=-k_{1} \tau .
$$

We will now use our solutions to extend the dispersion relation to general values of the flux parameter, beyond the WZW limit of the action. At these point we should emphasize

\footnotetext{
${ }^{3}$ We must note that when we move away from the limit of pure NS-NS flux the division of the solutions in three different regimes breaks down. However, we can still find $\tan t(\tau)$ in terms of complete elliptic integrals.
} 
that, despite we have chosen $\bar{F}_{1}$ and $\omega$ as constants of motion, any other set of first integrals is valid as long as they remain independent in phase space. In particular, we can replace $\bar{F}_{1}$ by $\bar{n}$ in the argument of the elliptic sine, $\operatorname{sn}(\bar{n} \tau, \nu)=\operatorname{sn}\left(\sqrt{\left(1-q^{2}\right)\left(\mu_{3}-\mu_{1}\right)} \tau, \nu\right)$. Thus

$$
\bar{n}^{4}=\left[k_{1}^{2}-\omega^{2}+2 q k_{1}\left(u_{0}-q k_{1}\right)\right]^{2}+4\left(1-q^{2}\right) k_{1}^{2}\left(u_{0}-q k_{1}\right)^{2} .
$$

When we combine this expression with the Virasoro constraint (28) and solve for $u_{0}$ we conclude that

$$
E=-\sqrt{\lambda} u_{0}=-\frac{\sqrt{\lambda}}{2}\left[2 q k_{1}+\frac{1}{k_{1}}\left(-q\left(k_{1}^{2}-\omega^{2}\right) \mp \sqrt{\bar{n}^{4}-\left(1-q^{2}\right)\left(k_{1}^{2}-\omega^{2}\right)}\right)\right],
$$

which in the limit of pure NS-NS flux becomes

$$
E=-\frac{\sqrt{\lambda}}{2}\left(k_{1}+\frac{\omega^{2} \mp n^{2}}{k_{1}}\right)
$$

where $n$ is just the value of $\bar{n}$ at the WZW point. The upper sign in this expression corresponds to the short string regime, while the lower one is the long string regime, in accordance with [48]. A natural question arising from this letter is the derivation of (43) by extending the analysis of the conformal field theory beyond the limit of the WZW model. It would also be interesting to investigate in more detail the relation between the WZW model and the reduction of the Neumann-Rosochatius system with pure NS-NS flux.

\section{Acknowledgments}

The work of R.H. is supported by grant FPA2014-54154-P and by BSCH-UCM through grant GR3/14-A 910770.

Open Access. This article is distributed under the terms of the Creative Commons Attribution License (CC-BY 4.0), which permits any use, distribution and reproduction in any medium, provided the original author(s) and source are credited.

\section{References}

[1] A. Babichenko, B. Stefański Jr. and K. Zarembo, Integrability and the $A d S_{3} / C F T_{2}$ correspondence, JHEP 03 (2010) 058 [arXiv: 0912.1723] [INSPIRE].

[2] K. Zarembo, Strings on Semisymmetric Superspaces, JHEP 05 (2010) 002 [arXiv: 1003.0465] [INSPIRE].

[3] K. Zarembo, Algebraic Curves for Integrable String Backgrounds, arXiv:1005.1342 [INSPIRE].

[4] O. Ohlsson Sax and B. Stefański Jr., Integrability, spin-chains and the $A d S_{3} / C F T_{2}$ correspondence, JHEP 08 (2011) 029 [arXiv:1106. 2558] [INSPIRE].

[5] N. Rughoonauth, P. Sundin and L. Wulff, Near BMN dynamics of the $A d S_{3} \times S^{3} \times S_{3} \times S_{1}$ superstring, JHEP 07 (2012) 159 [arXiv:1204.4742] [INSPIRE]. 
[6] O. Ohlsson Sax, B. Stefański Jr. and A. Torrielli, On the massless modes of the AdS $S_{3} / C F T_{2}$ integrable systems, JHEP 03 (2013) 109 [arXiv:1211.1952] [INSPIRE].

[7] C. Ahn and D. Bombardelli, Exact $S$-matrices for $A d S_{3} / C F T_{2}$, Int. J. Mod. Phys. A 28 (2013) 1350168 [arXiv:1211.4512] [INSPIRE].

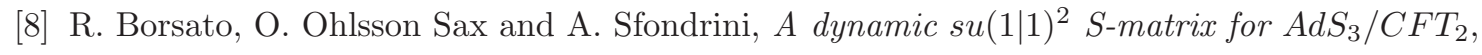
JHEP 04 (2013) 113 [arXiv:1211.5119] [INSPIRE].

[9] R. Borsato, O. Ohlsson Sax and A. Sfondrini, All-loop Bethe ansatz equations for $A d S_{3} / C F T_{2}, J H E P 04$ (2013) 116 [arXiv:1212.0505] [INSPIRE].

[10] R. Borsato, O. Ohlsson Sax, A. Sfondrini, B. Stefański Jr. and A. Torrielli, The all-loop integrable spin-chain for strings on $A d S_{3} \times S^{3} \times T^{4}$ : the massive sector, JHEP 08 (2013) 043 [arXiv: 1303.5995] [INSPIRE].

[11] R. Borsato, O. Ohlsson Sax, A. Sfondrini and B. Stefański Jr., Towards the All-Loop Worldsheet $S$ Matrix for $A d S_{3} \times S^{3} \times T^{4}$, Phys. Rev. Lett. 113 (2014) 131601 [arXiv: 1403.4543] [INSPIRE].

[12] R. Borsato, O. Ohlsson Sax, A. Sfondrini and B. Stefański Jr., The complete $A d S_{3} \times S^{3} \times T^{4}$ worldsheet $S$ matrix, JHEP 10 (2014) 066 [arXiv: 1406.0453] [INSPIRE].

[13] M. Beccaria, F. Levkovich-Maslyuk, G. Macorini and A.A. Tseytlin, Quantum corrections to spinning superstrings in $A d S_{3} \times S^{3} \times M^{4}$ : determining the dressing phase, JHEP 04 (2013) 006 [arXiv:1211.6090] [INSPIRE].

[14] M. Beccaria and G. Macorini, Quantum corrections to short folded superstring in $A d S_{3} \times S^{3} \times M^{4}, J H E P 03$ (2013) 040 [arXiv: 1212.5672] [INSPIRE].

[15] R. Borsato, O. Ohlsson Sax, A. Sfondrini, B. Stefański Jr. and A. Torrielli, Dressing phases of $A d S_{3} / C F T_{2}$, Phys. Rev. D 88 (2013) 066004 [arXiv: 1306.2512] [INSPIRE].

[16] M.C. Abbott, The $A d S_{3} \times S^{3} \times S^{3} \times S^{1}$ Hernández-López phases: a semiclassical derivation, J. Phys. A 46 (2013) 445401 [arXiv: 1306.5106] [inSPIRE].

[17] M.C. Abbott and I. Aniceto, Macroscopic (and Microscopic) Massless Modes, Nucl. Phys. B 894 (2015) 75 [arXiv:1412.6380] [INSPIRE].

[18] M.C. Abbott and I. Aniceto, An improved AFS phase for AdS $S_{3}$ string integrability, Phys. Lett. B 743 (2015) 61 [arXiv:1412.6863] [INSPIRE].

[19] P. Sundin and L. Wulff, Worldsheet scattering in $A d S_{3} / C F T_{2}$, JHEP 07 (2013) 007 [arXiv: 1302.5349] [INSPIRE].

[20] T. Lloyd and B. Stefański Jr., $A d S_{3} / C F T_{2}$, finite-gap equations and massless modes, JHEP 04 (2014) 179 [arXiv: 1312.3268] [INSPIRE].

[21] P. Sundin, Worldsheet two- and four-point functions at one loop in $A d S_{3} / C F T_{2}$, Phys. Lett. B 733 (2014) 134 [arXiv: 1403.1449] [InSPIRE].

[22] A. Sfondrini, Towards integrability for $A d S_{3} / C F T_{2}$, J. Phys. A 48 (2015) 023001 [arXiv: 1406.2971] [INSPIRE].

[23] A. Cagnazzo and K. Zarembo, B-field in $A d S_{3} / C F T_{2}$ Correspondence and Integrability, JHEP 11 (2012) 133 [Erratum JHEP 04 (2013) 003] [arXiv:1209.4049] [INSPIRE].

[24] B. Hoare and A.A. Tseytlin, On string theory on $A d S_{3} \times S^{3} \times T^{4}$ with mixed 3-form flux: tree-level S-matrix, Nucl. Phys. B 873 (2013) 682 [arXiv:1303.1037] [INSPIRE]. 
[25] B. Hoare and A.A. Tseytlin, Massive S-matrix of AdS $S_{3} \times S^{3} \times T^{4}$ superstring theory with mixed 3-form flux, Nucl. Phys. B 873 (2013) 395 [arXiv: 1304.4099] [InSPIRE].

[26] B. Hoare, A. Stepanchuk and A.A. Tseytlin, Giant magnon solution and dispersion relation in string theory in $A d S_{3} \times S^{3} \times T^{4}$ with mixed flux, Nucl. Phys. B 879 (2014) 318 [arXiv: 1311.1794] [INSPIRE].

[27] C. Ahn and P. Bozhilov, String solutions in $A d S_{3} \times S^{3} \times T^{4}$ with NS-NS B-field, Phys. Rev. D 90 (2014) 066010 [arXiv:1404.7644] [InSPIRE].

[28] J.R. David and A. Sadhukhan, Spinning strings and minimal surfaces in AdS 3 with mixed 3-form fluxes, JHEP 10 (2014) 049 [arXiv: 1405.2687] [INSPIRE].

[29] A. Banerjee, K.L. Panigrahi and P.M. Pradhan, Spiky strings on $A d S_{3} \times S^{3}$ with $N S-N S$ flux, Phys. Rev. D 90 (2014) 106006 [arXiv:1405.5497] [InSPIRE].

[30] A. Babichenko, A. Dekel and O. Ohlsson Sax, Finite-gap equations for strings on $A d S_{3} \times S^{3} \times T^{4}$ with mixed 3-form flux, JHEP 11 (2014) 122 [arXiv: 1405.6087] [INSPIRE].

[31] L. Bianchi and B. Hoare, $A d S_{3} \times S^{3} \times M^{4}$ string $S$-matrices from unitarity cuts, JHEP 08 (2014) 097 [arXiv: 1405.7947] [INSPIRE].

[32] R. Roiban, P. Sundin, A. Tseytlin and L. Wulff, The one-loop worldsheet S-matrix for the $A d S_{n} \times S^{n} \times T^{10-2 n}$ superstring, JHEP 08 (2014) 160 [arXiv: 1407.7883] [INSPIRE].

[33] T. Lloyd, O. Ohlsson Sax, A. Sfondrini and B. Stefański Jr., The complete worldsheet $S$ matrix of superstrings on $A d S_{3} \times S^{3} \times T^{4}$ with mixed three-form flux,

Nucl. Phys. B 891 (2015) 570 [arXiv:1410.0866] [INSPIRE].

[34] P. Sundin and L. Wulff, One- and two-loop checks for the $A d S_{3} \times S^{3} \times T^{4}$ superstring with mixed flux, J. Phys. A 48 (2015) 105402 [arXiv:1411.4662] [INSPIRE].

[35] A. Stepanchuk, String theory in $A d S_{3} \times S^{3} \times T^{4}$ with mixed flux: semiclassical and 1-loop phase in the S-matrix, J. Phys. A 48 (2015) 195401 [arXiv:1412.4764] [InSPIRE].

[36] R. Borsato, O. Ohlsson Sax, A. Sfondrini and B. Stefański Jr., The $A d S_{3} \times S^{3} \times S^{3} \times S^{1}$ worldsheet $S$ matrix, J. Phys. A 48 (2015) 415401 [arXiv:1506.00218] [INSPIRE].

[37] A. Banerjee, K.L. Panigrahi and M. Samal, A note on oscillating strings in $A d S_{3} \times S^{3}$ with mixed three-form fluxes, JHEP 11 (2015) 133 [arXiv:1508.03430] [INSPIRE].

[38] A. Banerjee and A. Sadhukhan, Multi-spike strings in AdS $S_{3}$ with mixed three-form fluxes, JHEP 05 (2016) 083 [arXiv:1512.01816] [INSPIRE].

[39] A. Banerjee, S. Biswas and R.R. Nayak, D1 string dynamics in curved backgrounds with fluxes, JHEP 04 (2016) 172 [arXiv:1601.06360] [INSPIRE].

[40] R. Borsato, O. Ohlsson Sax, A. Sfondrini, B. Stefański Jr., A. Torrielli and O. Ohlsson Sax, On the dressing factors, Bethe equations and Yangian symmetry of strings on $A d S_{3} \times S^{3} \times T^{4}, J$. Phys. A 50 (2017) 024004 [arXiv: 1607.00914] [inSPIRE].

[41] S.P. Barik, M. Khouchen, J. Klusoň and K.L. Panigrahi, $\operatorname{SL}(2, Z)$ invariant rotating $(m, n)$ strings in $A d S_{3} \times S^{3}$ with mixed flux, Eur. Phys. J. C 77 (2017) 298 [arXiv:1610.03402] [INSPIRE].

[42] S.P. Barik, K.L. Panigrahi and M. Samal, Perturbations of Pulsating Strings, arXiv: 1708.05202 [INSPIRE]. 
[43] R. Hernández and J.M. Nieto, Spinning strings in $A d S_{3} \times S^{3}$ with NS-NS flux, Nucl. Phys. B 888 (2014) 236 [Erratum ibid. B 895 (2015) 303] [arXiv:1407.7475] [INSPIRE].

[44] R. Hernández and J.M. Nieto, Elliptic solutions in the Neumann-Rosochatius system with mixed flux, Phys. Rev. D 91 (2015) 126006 [arXiv:1502.05203] [INSPIRE].

[45] G. Arutyunov, S. Frolov, J. Russo and A.A. Tseytlin, Spinning strings in $A d S_{5} \times S^{5}$ and integrable systems, Nucl. Phys. B 671 (2003) 3 [hep-th/0307191] [INSPIRE].

[46] G. Arutyunov, J. Russo and A.A. Tseytlin, Spinning strings in $A d S_{5} \times S^{5}$ : New integrable system relations, Phys. Rev. D 69 (2004) 086009 [hep-th/0311004] [INSPIRE].

[47] K. Uhlenbeck, Equivariant harmonic maps into spheres, Lect. Notes Math. 949 (1982) 39.

[48] J.M. Maldacena and H. Ooguri, Strings in $A d S_{3}$ and $\mathrm{SL}(2, \mathbb{R})$ WZW model. I: The Spectrum, J. Math. Phys. 42 (2001) 2929 [hep-th/0001053] [INSPIRE]. 